\title{
Extension of the modified Effective Medium Approach to nanocomposites with anisotropic thermal conductivities
}

\author{
Iorwerth O. Thomas and G. P. Srivastava \\ School of Physics, University of Exeter, \\ Stocker Road, Exeter EX4 4QL, United Kingdom
}

(Dated: August 15, 2018)

\begin{abstract}
An extended modification of the effective medium approach (emEMA) has been developed for the thermal conductivity of anisotropic nanocomposites. This is based on extending approaches developed to treat anisotropic particle insert and host matrix in electromagnetism of composites to anisotropic thermal interface resistance, with the inclusion of insert size and interface boundary density effects. The method has been applied to the case of spherical inclusions of the $2 \mathrm{H}$ dichalcodenide $\mathrm{WS}_{2}$ within a matrix of $2-\mathrm{H} \mathrm{MoS}_{2}$, with input bulk thermal conductivities calculated using our recently developed semi-ab initio method. We find that the overall effects of anisotropy are strongest for small particles, but that as particle size increases the effects of the surface anisotropy effects become more apparent.

PACS numbers:
\end{abstract}




\section{INTRODUCTION}

Effective medium approaches (EMAs) have long been applied to problems in electromagnetism, elasticity and thermal conductivity in order to account in a simple way for the effects of inclusions with a different value of the dielectric constant, stiffness or thermal conductivity from the surrounding matrix or background medium. Nan et al.'s seminal paper [1] derives a general EMA expression for thermal conductivity of a two-component composite in the presence of interface (Kapitza) resistance that can be shown to reduce to various previously derived special cases. Palla and Giordano [2] have examined particles with anisotropic conductivity and isotropic boundary resistances embedded within isotropically conducting matrices using a similar scheme, and Gavalas [3] has examined spherical core-shell systems with finite shell-size. Minnich and Chen [4] have proposed a modified effective medium approach (mEMA) which incorporates the effects of interface boundary density and insert size for spherical particles, providing better agreement with Monte Carlo simulations. Ordonez-Miranda et al. [5] and Behrang et al. [6-8] have further examined the effects of differing insert shapes and interface roughness within the mEMA.

However, the basic formulae on which both methods (EMA and mEMA) depend are derived on the assumption that the thermal (or electrical) conductivity of the matrix medium is isotropic. This is not true of materials in general: for example, graphene and transition metal dichalcogenides (TMDs) possess anisotropic thermal conductivities due to their layered structures, and if they are used as a matrix for particle insertions to produce composite structures, this must be taken into account in order to obtain the correct solution to the Laplace's equation on which both EMA and mEMA rely. For the electromagnetic EMA, Sihvola [9] has derived a generalised form of the Maxwell-Garnett equation that is valid for anisotropic external dielectric tensors (see also the discussion and references within Ref. [10]). For the thermal conductivity EMA recent publications examine the problem rigorously within multipole [11] and micromechanical [12] frameworks, but as far as the authors of are aware the effects of matrix anisotropy within the mEMA have not yet been examined.

In this work, we examine the extension of the basic anisotropic EMA of Sihvola [9] to a Minnich and Chen-type mEMA [4] for spherical inclusions within an anisotropic thermally conducting matrix, using a generalisation of Nan et al.'s basic approach [1] to account for anisotropic Kapitza resistances. We use this extended modification of the effective medium 
approach (emEMA) to examine the effects of including anisotropy for the case of spherical inclusions of the 2-H TMD $\mathrm{WS}_{2}$ within a matrix of $\mathrm{MoS}_{2}$, where the input thermal conductivities have been calculated using a previously described semi-ab initio method $[13,14]$. We find that the anisotropies present in the system have a noticable effect on the results of the calculation.

\section{THEORY}

Following Nan et al. [1] we consider a system consisting of a matrix with a thermal conductivity tensor $\kappa^{m}$, an insert with thermal conductivity tensor $\kappa^{i}$ and a surface layer that surrounds the insert and separates it from the matrix with thermal conductivity tensor $\kappa^{s}$. If either one (or both) of the matrix or insert exhibit an anisotropic thermal conductivity (i.e. at least one of the $x x, y y$ and $z z$ components differs from the others), then since the surface region can be thought of as a mixture of the matrix and insulating region it is reasonable to assume that it will also exhibit anisotropy, and that this will persist when we come to consider the Kapitza resistance of the surface region in the limit where it is thin and poorly conducting. That is, for a spherical insert, the heat flux normal to the interface (which is not generally parallel to the $x, y$ or $z$ axis) could be decomposed into different $x$, $y, z$ components, implying a directionally dependent (i.e. anisotropic) surface conductance and hence resistance. In order to correctly calculate the effective thermal conductivity of the insert and its surface together, we must perform an affine transformation that restores the isotropy of the surface, resulting in an effective distortion of the insert shape $[9,10]$.

We begin with Sihvola's generalisation [9] of the Maxwell-Garnett equation, rewritten for the case of thermal conductivities $\kappa^{s}$ and $\kappa^{i}$ :

$$
\begin{aligned}
\kappa^{*} & =\kappa^{s}+v\left(\kappa^{i}-\kappa^{s}\right) \mathbb{L}^{-1}, \\
\mathbb{L} & =\mathbb{I}+(1-v) \mathbb{N}_{s}\left(\kappa^{i}-\kappa^{s}\right)\left(\kappa^{s}\right)^{-1},
\end{aligned}
$$

where the thermal conductivity tensors, $\mathbb{L}, \mathbb{N}_{s}$ and $\mathbb{I}$ are all $3 \times 3$ matrices. $\kappa^{*}$ is the effective thermal conductivity tensor for the surface and insert considered together, $\mathbb{I}$ is the identity matrix, $\mathbb{N}_{s}$ is the geometry dependent depolarisation tensor for the system following the affine transformation required to solve Laplace's equation in the presence of anisotropy $[9,10]$, and $v=a^{3} /(a+\delta)^{3}$ where $a$ is the radius of a spherical insert and $\delta$ the thickness of the surface layer. 
We assume that the system is oriented such that $\kappa^{s}$ is diagonal and take the limits $\delta \rightarrow 0$ and $\kappa^{s} \rightarrow 0$. The anisotropic Kapitza resistance is defined as follows:

$$
\mathbb{R}^{K}=\left(\begin{array}{ccc}
\lim _{\substack{\delta \rightarrow 0 \\
\kappa_{x x}^{s} \rightarrow 0}}\left(\frac{\delta}{\kappa_{x x}^{s}}\right) & 0 & 0 \\
0 & \lim _{\substack{\delta \rightarrow 0 \\
\kappa_{y y}^{s} \rightarrow 0}}\left(\frac{\delta}{\kappa_{y y}^{s}}\right) & 0 \\
0 & 0 & \lim _{\substack{\delta \rightarrow 0 \\
\kappa_{z z}^{s} \rightarrow 0}}\left(\frac{\delta}{\kappa_{z z}^{s}}\right)
\end{array}\right),
$$

where the limits are taken such that

$$
\frac{\mathbb{R}_{x x}^{K}}{\mathbb{R}_{z z}^{K}}=\lim _{\substack{\kappa_{z z}^{s} \rightarrow 0 \\ \kappa_{x x}^{s} \rightarrow 0}}\left(\frac{\kappa_{z z}^{s}}{\kappa_{x x}^{s}}\right), \quad \frac{\mathbb{R}_{y y}^{K}}{\mathbb{R}_{z z}^{K}}=\lim _{\substack{\kappa_{z z}^{s} \rightarrow 0 \\ \kappa_{y y}^{s} \rightarrow 0}}\left(\frac{\kappa_{z z}^{s}}{\kappa_{y y}^{s}}\right) .
$$

From this we may recover a generalised form of the EMA equation [1] of a spherical insert in the presence of surface resistance:

$$
\kappa^{*}=\kappa^{i}\left[\mathbb{I}+\frac{3}{a} \mathbb{N}_{s} \kappa^{i} \mathbb{R}^{K}\right]^{-1} .
$$

In the case where the $\kappa^{s}$ is isotropic, $\mathbb{N}_{s}=\frac{1}{3} \mathbb{I}$ and equation (7) of Ref. [1] may be recovered for the case of a spherical insert.

In order to obtain the overall effective thermal conductivity tensor $\kappa^{E}$ for the insert-matrix composite, we utilise Sihovola's generalised equation in its unaltered form [9], assuming that our coordinate axes are such that $\kappa^{m}$ is diagonal:

$$
\begin{aligned}
\kappa^{E} & =\kappa^{m}+f\left(\kappa^{*}-\kappa^{m}\right) \mathbb{J}^{-1} \kappa^{m}, \\
\mathbb{J} & =\kappa^{m}+(1-f) \mathbb{N}_{m}\left(\kappa^{i}-\kappa^{m}\right) .
\end{aligned}
$$

Here, $f$ is the volume fraction of inserts within the matrix, and $\mathbb{N}_{m}$ is the depolarisation tensor following the affine transformation with respect to the anisotropy of the matrix (this is different from $\mathbb{N}_{s}$, which is the depolarisation tensor due to the anisotropy of the interface/surface). We have not attempted to derive a closed form expression here, but this approach should give indentical results and is readily generalisable to more complex nanocomposites.

Expressions for the depolarisation tensor in different cases may be found in (for example) Ref. [15]. For the system examined in this study we will need only the definitions for the 
case of prolate spheroids. Firstly, we define affine transformations $[9,10]$ :

$$
\begin{aligned}
& a_{s, x}=\sqrt{\frac{\mathbb{R}_{x x}^{K}}{\mathbb{R}_{z z}^{K}}} a, \quad a_{s, y}=\sqrt{\frac{\mathbb{R}_{y y}^{K}}{\mathbb{R}_{z z}^{K}}} a, \quad a_{s, z}=a, \\
& a_{m, x}=\sqrt{\frac{\kappa_{z z}^{s}}{\kappa_{x x}^{s}}} a, \quad a_{m, y}=\sqrt{\frac{\kappa_{z z}^{s}}{\kappa_{y y}^{s}}} a, \quad a_{m, z}=a,
\end{aligned}
$$

where we have used the relationship between resistance and conductivity to obtain an effective thermal conductivity anisotropy in the case of the surface. Assuming that $a_{l, z}>a_{l, x}=a_{l, y}$ for $l=s, m$, and that the system is oriented so that $\kappa^{l}$ is diagonal for its respective mixing step, $\mathbb{N}_{l}$ will be a diagonal matrix with non-zero components [15]:

$$
\begin{aligned}
& \mathbb{N}_{l, z z}=\frac{1-e^{2}}{2 e^{3}}\left(\ln \frac{1+e}{1-e}-2 e\right), \text { where } e=\sqrt{1-\frac{a_{l, x}^{2}}{a_{l, z}^{2}}}, \\
& \mathbb{N}_{l, x x}=\mathbb{N}_{l, y y}=\frac{1}{2}\left(1-\mathbb{N}_{l, z z}\right) .
\end{aligned}
$$

The final ingredient of our approach is the modification of $\kappa^{i}$ and $\kappa^{m}$ in accordance with the mEMA approach outlined in Ref. [4]. The method of calculation is outlined in the Supplementary Information. In brief, we obtain $\kappa^{i}$ and $\kappa^{m}$ using our recently developed semi- $a b$ inito theory [13] based on the single-mode relaxation time approximation for the linearized phonon transport equation. $\kappa^{i}$ is calculated in the usual fashion but with the insert boundary scattering length $L_{B}$ set equal to $L_{B, I}=2 a$ (i.e. the diameter of the insert), whereas $\kappa^{m}$ aquires an additional scattering length $L_{I S}=4 / \Phi$ representing scattering from insertion interfaces. Here $\Phi=6 f / L_{B, I}$ represents the interface density of the composite at a given volume fraction $f$ and insert size $2 a$. We calculate the thermal boundary resistance matrix $R_{T B R}$ using a generalised form of Chen's expression [4, 16], but with the modification $v_{\|}=\sqrt{v_{x}^{2}+v_{y}^{2}}$ and $v_{\perp}=\left|v_{z}\right|$ for the speed of a given phonon mode when considering the anisotropic case:

$$
R_{T B R}=4\left(\frac{\left\langle C_{1} v_{1}\right\rangle+\left\langle C_{2} v_{2}\right\rangle}{\left\langle C_{1} v_{1}\right\rangle\left\langle C_{2} v_{2}\right\rangle}\right)
$$

Here, 1 and 2 label the matrix and the insert respectively, and $\left\langle C_{i} v_{i}\right\rangle=\sum_{\boldsymbol{q} s} C_{i, \boldsymbol{q} s} v_{i, \boldsymbol{q} s}$, with $C_{i, \boldsymbol{q} s}$ being the specific heat contribution from mode $\boldsymbol{q} s$ for material $i$. Note that this expression is an approximation since the original form is derived for scattering from a superlattice, in which it will tend to overestimate the value of the thermal boundary resistance. Accurate computation of this quantity is not straightforward, and may require a detailed ab-initio approach (eg. Ref. [17]). 


\section{RESULTS AND DISCUSSION}

As a sample application of the method, we consider nanocomposites of the dichalcogenides $\mathrm{MoS}_{2}$ and $\mathrm{WS}_{2}$ in their $2 \mathrm{H}$ bulk form. Specifically, we model spherical inserts of $\mathrm{WS}_{2}$ in a matrix of $\mathrm{MoS}_{2}$ (sample size $L_{B, M}=10 \mu \mathrm{m}$ ), where the cross-plane conductivity components of both are taken to be parallel to each other. We discuss the method used to calculate the thermal conductivities for each material in the Appendix.

Figure 1 compares the isotropic $R_{T B R}$ with the anisotropic $R_{T B R}$. For both cases the resistances decrease with increase in $T$ towards an asymptotic value, saturating at around $T=500 \mathrm{~K}$. The in-plane ( $x$ or $y$ direction) anisotropic $R_{T B R}$ is virtually identical to the isotropic $R_{T B R}$ since the in-plane velocity components make up the bulk of the contribution to the isotropic average velocities, whereas the cross-plane ( $z$ direction) anistropic $R_{T B R}$ is different, saturating at a value that is an order of magnitude larger. This is consistent with what we would expect of a layered material - the frequency dispersion of phonons in the cross-plane direction is much lower than in the planar direction, thus lowering the thermal conductivity in that direction and so leading to a larger thermal resistance.

In Figures 2 and 3 the legend 'All isotropic' refers to calculations carried out using the isotropic mEMA formalism using the anisotropic thermal conductivities, and we compare the effects of the inclusions of different levels of anisotropy with this uncorrected result.

The effects of anisotropic thermal resistance for two different concentrations of $10 \mathrm{~nm}$ and $1000 \mathrm{~nm}(1 \mu \mathrm{m})$ spherical inserts can be seen in Figure 2. Calculations using anisotropic $R_{T B R}$ and isotropic EMA formulae leave the in-plane $\kappa_{x x, y y}$ unchanged while lowering the thermal conductivity $\kappa_{z z}$ in the cross-plane direction. (This is most obvious in Figure 2 (b) and (c).) Typically, the inclusion of the effects of surface and matrix thermal conductivity counter-act this by lowering the in-plane thermal conductivity and increasing the cross-plane thermal conductivity. In both directions the thermal conductivity calculated for an isotropic $R_{T B R}$ while including the effects of the anisotropic matrix is larger than that calculated with all anisotropic effects included, but for the $10 \mathrm{~nm}$ inserts the fully anisotropic results are closer in value to it than for the $1000 \mathrm{~nm}$ inserts. This is to be expected since the effect of $R_{T B R}$ on the effective thermal conductivity should be smaller for smaller-sized inserts, and the effect of internal and external scattering from insert interfaces introduced in the mEMA become more important in those cases. 
In order to facilitate comparisons between different levels of theory we also present in each panel of Figure 2 the ratio of in-plane and cross-plane conductivities. We find that $\kappa_{x x, y y} / \kappa_{z z}$ (anisotropic $R_{T B R}$ only) $>\kappa_{x x, y y} / \kappa_{z z}$ (isotropic) $>$ $\kappa_{x x, y y} / \kappa_{z z}$ (anisotropic matrix only) $>\kappa_{x x, y y} / \kappa_{z z}$ (all anisotropies). Increasing the volume fraction $f$ increases the range between the highest and lowest values of the ratio for each insert size. Decreasing the insert size while keeping $f$ constant also increases this range. However, the difference between $\kappa_{x x, y y} / \kappa_{z z}$ (anisotropic matrix only) and $\kappa_{x x, y y} / \kappa_{z z}$ (all anisotropies) is only significant for the $1000 \mathrm{~nm}$ insert. This provides a good measure of the effect of the surface contribution; we can see for the smaller particle it is negligible, but for the larger particle it will reduce the ratio of the conductivities further than might be expected from consideration of the anisotropy of the matrix alone.

Figure 3 displays the behaviour of the conductivity ratio $\kappa_{x x, y y} / \kappa_{z z}$ for three insert sizes at $T=300 \mathrm{~K}$ as the volume fraction $f$ is increased from 0.08 to 0.5 . It is clear that not only does the anisotropy increase as the particle size is decreased, but that the size of the overall correction due to anisotropy also increases. However, the effect of the surface anisotropy decreases with decrease in particle size. This suggests that while one can in principle ignore surface effects for very small $f$ or insert sizes in an mEMA model, the matrix anisotropy cannot be so ignored. We also note that the behaviour of $\kappa_{x x, y y} / \kappa_{z z}$ as $f$ is increased is sample size dependent: 10 and $100 \mathrm{~nm}$ inserts reach a different maximum value before decreasing at different rates, while the $1000 \mathrm{~nm}$ inserts increase monotonically. Note that the emEMA approach is at best valid for $f$ less than $\approx 0.2$. Beyond this point the effects of insert-insert interactions must be considered, and so the regions of the figure where we begin to see different sample sizes exhibit similar conductivity ratios are at this point somewhat speculative.

As the percolation threshold is reached, one would expect a sudden jump in the thermal conductivity. This is not just a result of the isolated insert assumption of the EMA formalism becoming invalid at the transition, but because the clusters of inserts connecting one side of the sample to the other will suddenly have a different, longer effective scattering length from the isolated inserts considered in the mEMA. As a result the value of $\kappa^{i}$ considered must sharply increase. It is difficult to predict the effect of the surface and matrix anisotropies in this regime, not only because the peturbation theory we have relied on has broken down but because the extended clusters of inserts could be considered to be single objects with 
overall depolarisation tensors very different from those of spheroids. One might speculate from the trends seen in Figure 3 that because the surface area of a given cluster is large, both the its and the matrix anisotropy might be expected to have a notable effect, reducing $\kappa_{x x, y y} / \kappa_{z z}$ well below the value predicted using a wholly isotropic formalism. This remains to be verified, however.

\section{CONCLUSION}

In summary, we have derived a formalism that accounts for the effects of anisotropic thermal boundary resistance within nano-composites whose components have anisotropic thermal conductivities. We have considered a simple example of such a system and shown that surface layer anisotropic thermal conductivity effects can be seen for spherical inserts of $1000 \mathrm{~nm}$ size, and that the effects of the overall matrix anisotropy should not be neglected for smaller inserts down to $10 \mathrm{~nm}$ size. Note that in this work we have considered one of the simplest possible geometries together with the simplest possible particle orientation, and that different orientations, different boundary resistence models and different geometries within the emEMA could display more profound effects as a result of thermal conductivity anisotropy.

\section{Acknowledgement}

This work was supported by Leverhulme Trust (UK) Grant No. RPG-2016-186. Calculations were performed on the Ceres cluster at the University of Exeter.

\section{Appendix}

\section{A. Calculation of the thermal conductivity}

We work within the single-mode relaxation time [18] approximation. The thermal conductivity tensor is written as:

$$
\kappa_{i j}=\frac{\hbar^{2}}{N_{0} \Omega k_{B} T^{2}} \sum_{\boldsymbol{q} s} \omega^{2}(\boldsymbol{q} s) v_{s}^{i}(\boldsymbol{q}) v_{s}^{j}(\boldsymbol{q}) \tau_{\boldsymbol{q} s} \bar{n}(\boldsymbol{q} s)(\bar{n}(\boldsymbol{q} s)+1) .
$$


Here, $\Omega$ is the volume of each unit cell and $N_{0}$ their number, $\omega(\boldsymbol{q} s)$ the frequency of a phonon mode with wavevector $\boldsymbol{q}$ and polarisation $s, v_{s}^{i}(\boldsymbol{q})$ is the corresponding group velocity component and we denote the Bose-Einstein distribution function with $\bar{n}(\boldsymbol{q} s) . \tau_{\boldsymbol{q} s}$ is the single mode relaxation time expressed as the inverse of the sum of the following scattering rates

$$
\begin{gathered}
\tau_{\boldsymbol{q}^{s}}^{-1}=\tau_{\boldsymbol{q}^{s}}^{-1}(\mathrm{bulk})+\tau_{\boldsymbol{q}^{s}}^{-1}(\mathrm{coll}), \\
\tau_{\boldsymbol{q} s}^{-1}(\mathrm{bulk})=\tau_{\boldsymbol{q} s}^{-1}(\mathrm{md})+\tau_{\boldsymbol{q} s, N}^{-1}+\tau_{\boldsymbol{q} s, U}^{-1},
\end{gathered}
$$

where $\tau_{\boldsymbol{q}^{s}}^{-1}$ (bulk) are the contributions independent of scattering from sample or interface boundaries (isotope mass-defect, Normal anharmonic and Umklapp anharmonic respectively) and $\tau_{\boldsymbol{q}_{\boldsymbol{s}}}^{-1}$ (coll) gives the contributions arising from interface and boundary scattering as described in the mEMA model.

Note that the single-mode relaxation time approximation typically gives a lower bound for the thermal conductivity in 2D and quasi-2D systems [19] and is being used here for its relative simplicity since our concern is the qualitative effect of surface and matrix anisotropies on the effective medium. More accurate calculations that include effects ignored by the single-mode relaxation time approximation may be performed using the Callaway [18, 20, 21] or iterative [22-24] approaches.

\section{Contributions independent of sample or interface scattering}

We obtain the isotopic mass-defect contribution to the total scattering rate from the expression [18]

$$
\tau_{\boldsymbol{q} s}^{-1}(\mathrm{md})=\frac{\pi}{2 N_{0}} \omega_{\boldsymbol{q} s}^{2} \sum_{\boldsymbol{q}^{\prime} s^{\prime}} \delta\left(\omega_{\boldsymbol{q} s}-\omega_{\boldsymbol{q}^{\prime} s^{\prime}}\right) \sum_{\boldsymbol{b}} \Gamma_{\mathrm{md}}(\boldsymbol{b})\left|\boldsymbol{e}_{\boldsymbol{q} s}^{\star}(\boldsymbol{b}) \cdot \boldsymbol{e}_{\boldsymbol{q}^{\prime} s^{\prime}}(\boldsymbol{b})\right|^{2},
$$

where $\boldsymbol{b}$ labels the site of an atom in the unit cell, the eigenvectors of the lattice dynamical matrix are given by $\boldsymbol{e}_{\boldsymbol{q} s}(\boldsymbol{b})$ and we compute the mass disorder coefficient $\Gamma_{\mathrm{md}}(\boldsymbol{b})$ for atom $b$ using

$$
\Gamma_{\mathrm{md}}(\boldsymbol{b})=\sum_{i} f_{i}(\boldsymbol{b})\left[1-M_{i}(\boldsymbol{b}) / \bar{M}(\boldsymbol{b})\right]^{2} .
$$

Here, $\bar{M}(\boldsymbol{b})$ is the average mass of the $\mathrm{b}^{\text {th }}$ atom and $f_{i}(\boldsymbol{b})$ is the proportion of the $i^{\text {th }}$ isotope that has mass $M_{i}(\boldsymbol{b})$. 
We use our semi-ab initio scheme [13] to calculate the anharmonic contribution arising from three-phonon processes [18]:

$$
\begin{aligned}
\tau_{3 \mathrm{ph}, \boldsymbol{q} s}^{-1} & =\frac{\pi \hbar}{\varrho N_{0} \Omega} \frac{\overline{\gamma^{2}}(T)}{\bar{c}^{2}} \sum_{\boldsymbol{q}^{\prime} s^{\prime}, \boldsymbol{q}^{\prime \prime} s^{\prime \prime}, \boldsymbol{G}} \omega \omega^{\prime} \omega^{\prime \prime} \delta_{\boldsymbol{q}+\boldsymbol{q}^{\prime}+\boldsymbol{q}^{\prime \prime}, \boldsymbol{G}} \\
& \times\left[\frac{\bar{n}^{\prime}\left(\bar{n}^{\prime \prime}+1\right)}{(\bar{n}+1)} \delta\left(\omega+\omega^{\prime}-\omega^{\prime \prime}\right)+\frac{1}{2} \frac{\bar{n}^{\prime} \bar{n}^{\prime \prime}}{\bar{n}} \delta\left(\omega-\omega^{\prime}-\omega^{\prime \prime}\right)\right],
\end{aligned}
$$

where we have suppressed the wavevector and polarisation indices for $\omega$ and $\bar{n}$. Here, $\bar{c}$ is the average acoustic velocity, $\varrho$ is the mass density and $\overline{\gamma^{2}}(T)$ is the mode-averaged, temperature dependent squared Grüneisen parameter calculated within the quasi-harmonic approximation [18, 25-27]. $\boldsymbol{G}=0$ indicates Normal processes, $\boldsymbol{G} \neq 0$ indicates Umklapp processes.

\section{Sample and interface scattering contributions within the mEMA model}

As noted in the main text, we account for interface and sample boundary scattering in the insert and in the matrix differently in accordance with the directions of Minnich et al [4]:

$$
\begin{aligned}
\tau_{\boldsymbol{q} s}^{-1}(\text { coll }) & =\frac{\left|v_{s}(\boldsymbol{q})\right|}{L_{B}}, \\
\text { where } L_{B}^{-1} & = \begin{cases}L_{B, I}^{-1} & \text { for inserts } \\
L_{B, S}^{-1}+L_{I S}^{-1} & \text { for the matrix }\end{cases}
\end{aligned}
$$

where $L_{B, I}$ is the diameter of the insert, $L_{B, S}$ is the size of the matrix sample $(10 \mu \mathrm{m}$ in this case) and $L_{I S}$ is the effective scattering length arising from the presence of interfaces in the matrix. These are described more fully in the main text.

\section{B. Generation of phonon eigensolutions using density functional theory}

Phonon force constants were generated ab initio for bulk $\mathrm{MoS}_{2}$ and $\mathrm{WS}_{2}$ using the Quantum Espresso package [28] for $8 \times 8 \times 2$ Monkhorst-Pack (MP) [29] grids and PBE [30] pseudopotentials. From these, frequencies, eigenvectors and velocities were generated for 
$28 \times 28 \times 7 \mathrm{MP}$ grids. For full details of these calculations, see Ref. [14].

[1] C.-.W Nan, R. Birringer, D. R. Clarke, and H. Gleiter, J. Appl. Phys. 81, 6692 (1997).

[2] P. L. Palla and S. Giordano, J. Appl. Phys. 120, 184301 (2016).

[3] G. R. Gavelas, AIP Advances 7, 095222 (2017).

[4] A. Minnich and G. Chen, Appl. Phys. Lett. 91, 073105 (2007).

[5] J. Ordonez-Miranda, R. Yang, and J. J. Alvarado-Gil, Appl. Phys. Lett. 98, 233111 (2011).

[6] A. Behrang, M. Grmela, C. Dubois, S. Turenne, and P. G. Lafleur, J. Appl. Phys. 114, 014305 (2013).

[7] A. Behrang, M. Grmela, C. Dubois, S. Turenne, P. G. Lafleur, and G. Lebon, Appl. Phys. Lett. 104, 063106 (2014).

[8] A. Behrang, M. Grmela, C. Dubois, S. Turenne, and P. G. Lafleur, RSC Adv. 5, 2768 (2015).

[9] A. Sihvola, Electromagnetics 17, 269 (1997).

[10] O. Levy and E. Cherkaev, J. Appl. Phys. 114, 164102 (2013).

[11] V. I. Kushch, I. Sevostianov, and A. Giraud, Proc. R. Soc A 473, 0472 (2018).

[12] S. Lee, J. Lee, B. Ryu, and S. Ryu, Scientific Reports 8, 7266 (2018).

[13] I. O. Thomas and G. P. Srivastava, J. Phys.: Condens. Matter 29, 505703 (2017).

[14] I. O. Thomas and G. P. Srivastava, J. App. Phys 123, 13135703 (2018).

[15] A. Sihvola, Electromagnetic mixing formulas and applications (The Institute of Electrical Engineers, London, 1999).

[16] G. Chen, Phys. Rev. B 57, 14958 (1998).

[17] A. Alkurdi, S. Pailhès and S. Merabia, Applied. Phys. Lett. 111, 093101 (2011).

[18] G. P. Srivastava, The Physics of Phonons (Taylor and Francis: New York, 1990).

[19] J. Ma, W. Li, and X. Luo, Phys. Rev. B 90, 035203 (2014).

[20] J. Callaway, Phys. Rev. 113, 1046 (1959).

[21] G. P. Srivastava, Rep. Prog. Phys. 78, 026501 (2015).

[22] M. Omini and A. Sparavigna, Physica B 212, 101 (1995).

[23] M. Omini and A. Sparavigna, Phys. Rev. B 53, 9064 (1996).

[24] D. A. Broido, A. Ward, and N. Mingo, Phys. Rev. B 72, 014308 (2005).

[25] L. Bjerg, B. B. Iverson and G. K. Madsen, Phys. Rev B 89, 024304 (2014). 
[26] G. H. K. Madsen, A. Katre and C. Bera, Phys. Status Solidi A 215, 802 (2016).

[27] A. Katre and G. K. H. Madsen, Phys. Rev. B 93, 155203 (2016).

[28] P. Gianozzi et al, J. Phys. Cond. Mat. 21, 395502 (2009); code available from http://www.quantum-espresso.org.

[29] H. J. Monkhorst and J. D. Pack, Phys. Rev. B 13, 5188 (1976).

[30] P. Perdew, K. Burke and M. Ernzerhof, Phys. Rev. Lett. 77, 3865 (1996). 


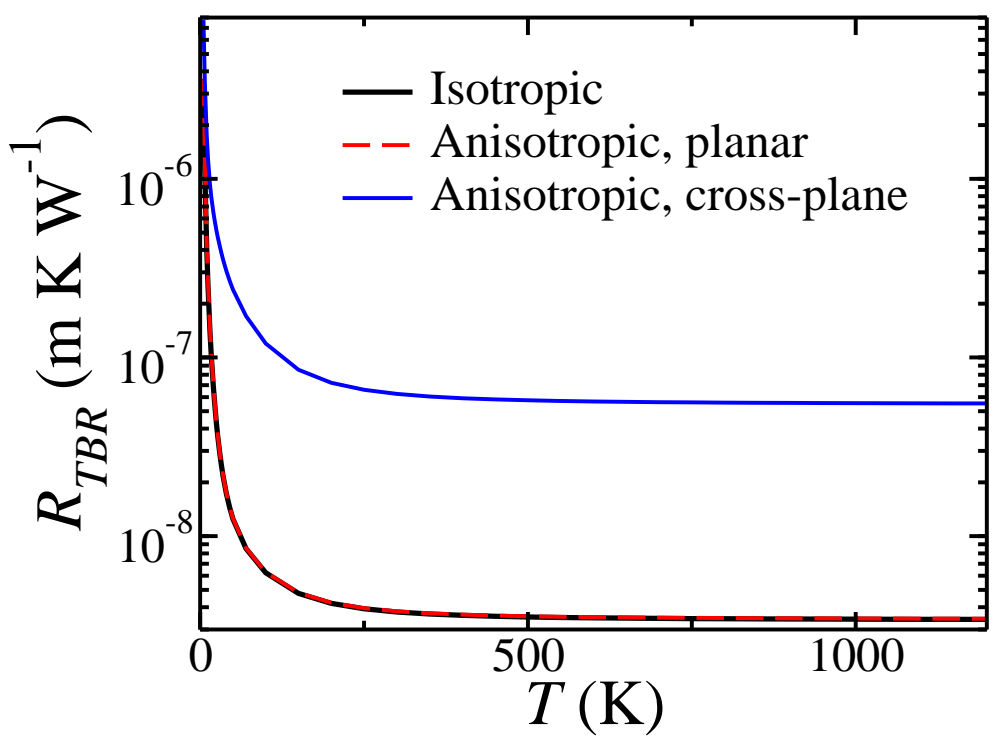

FIG. 1: Comparison of isotropic and anisotropic thermal boundary resistivities as temperature is varied for a $2 \mathrm{H} \mathrm{WS} / \mathrm{MoS}_{2}$ composite structure. 

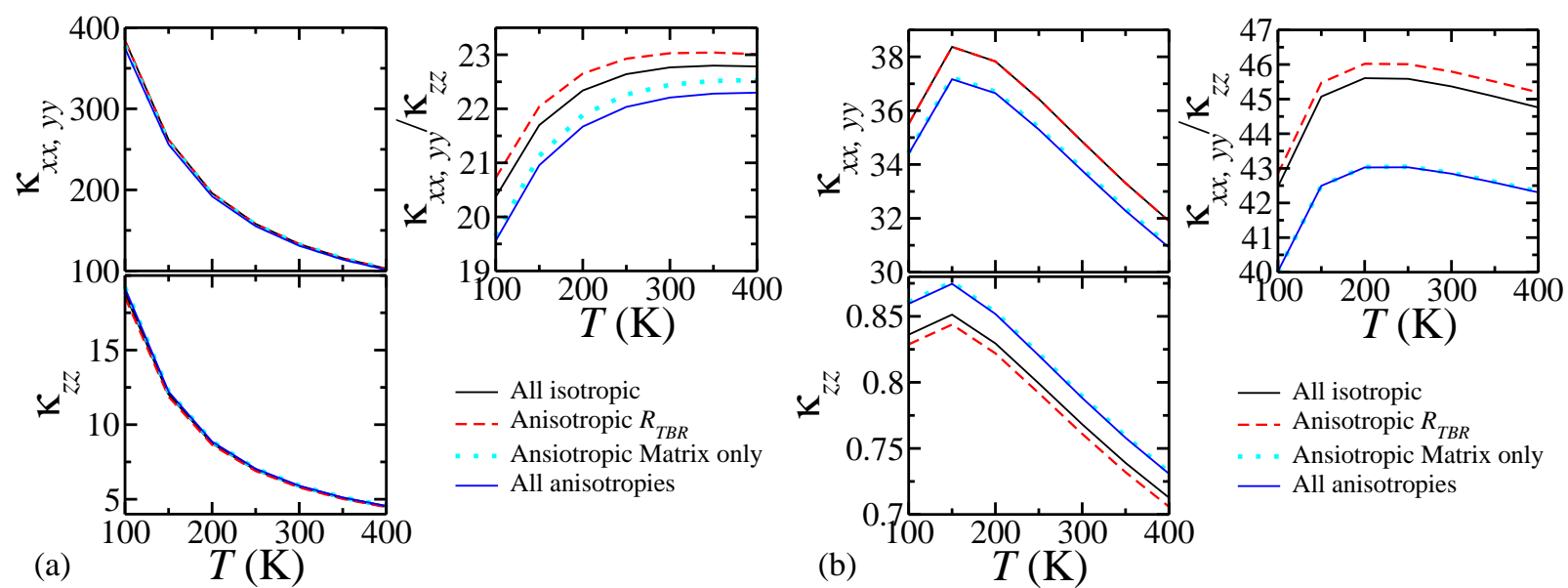

- All isotropic

- - - Anisotropic $R_{T B R}$

.. Ansiotropic Matrix only

— All anisotropies
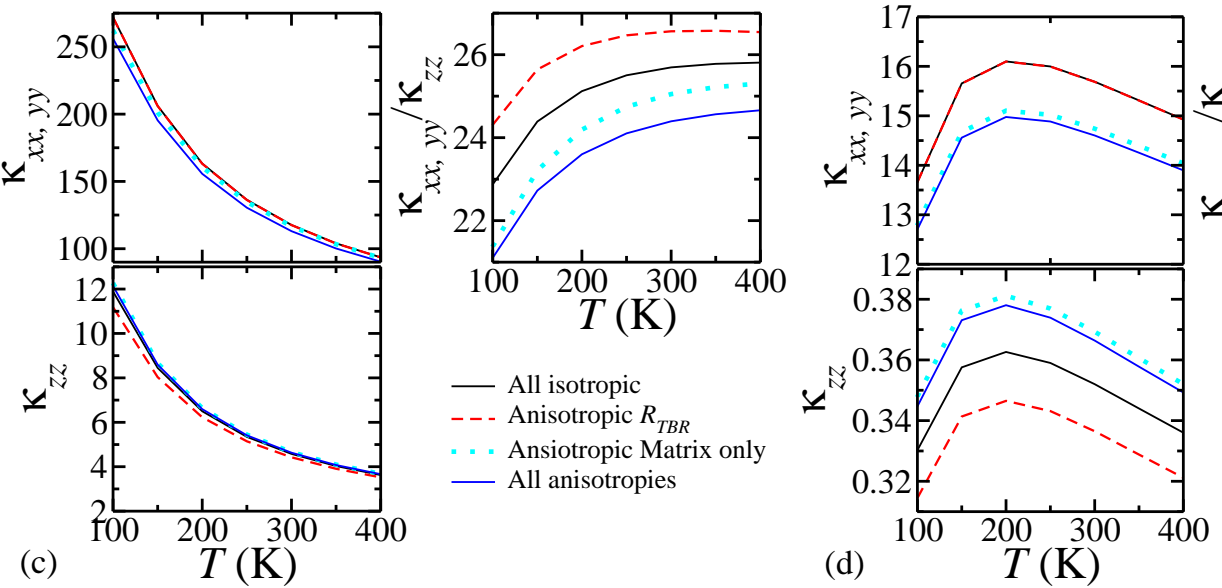

- All isotropic

- - - Anisotropic $R_{T B R}$

. - Ansiotropic Matrix only

_ All anisotropies

- All isotropic

- - - Anisotropic $R_{T B R}$

- = Ansiotropic Matrix only

_ All anisotropies

(c)

$T(\mathrm{~K})$

d)

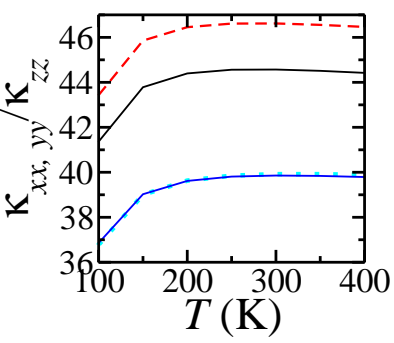

FIG. 2: Behaviour of the crossplane $\kappa_{z z}$ and in-plane thermal conductivities $\kappa_{x x, y y}$ in units of W $\mathrm{m}^{-1} \mathrm{~K}^{-1}$ and their ratio with temperature for $\mathrm{WS}_{2}$ inserts in a $\mathrm{MoS}_{2}$ matrix. (a) and (b): comparisons of $L_{B, I}=1000 \mathrm{~nm}$ and $L_{B, I}=10 \mathrm{~nm}$ respectively for $f=0.08$; (c) and (d) comparisons of $L_{B, I}=1000 \mathrm{~nm}$ and $L_{B, I}=10 \mathrm{~nm}$ respectively for $f=0.2$. 


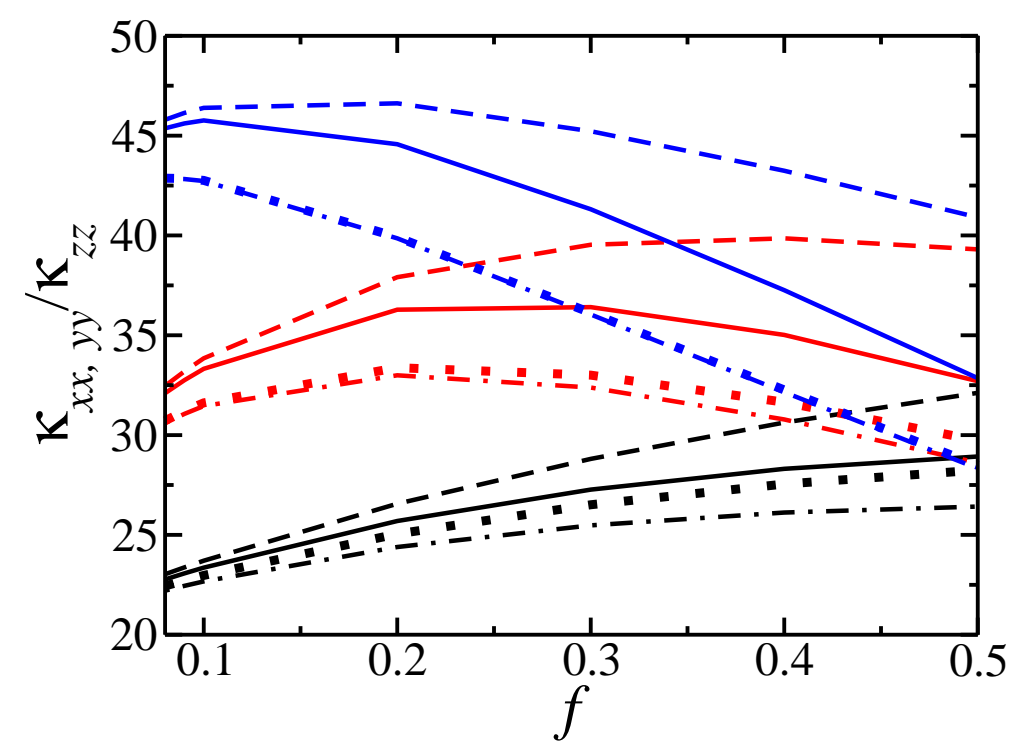

FIG. 3: Behavior of the ratio of cross and in-plane thermal conductivities with respect to volume fraction $f$ for $\mathrm{WS}_{2}$ inserts in a $\mathrm{MoS}_{2}$ matrix. Black labels $1000 \mathrm{~nm}$ inserts, red $100 \mathrm{~nm}$ inserts, blue $10 \mathrm{~nm}$ inserts. Full lines respresent fully isotropic formalism; dashed lines include only anisotropic thermal boundary resistance and no more; dotted lines include matrix anisotropy only; and dotdashed lines show the full calculation (i.e. anisotropic thermal boundary resistance, effects of matrix and surface anisotropy). 